\title{
An Efficient Methodology for the Synthesis of 3-Styryl Coumarins
}

\author{
Sérgio M. A. Martins, ${ }^{a}$ Paula C. S. Branco ${ }^{b}$ and António M. D. R. L. Pereira ${ }^{*, a}$ \\ ${ }^{a}$ Centro de Química, Departamento de Química, Universidade de Évora, \\ Rua Romão Ramalho, 59, 7000-671 Évora, Portugal \\ ${ }^{b}$ REQUIMTE, Departamento de Química, Faculdade de Ciências e Tecnologia (FCT), \\ Universidade Nova de Lisboa, 2829-516 Caparica, Portugal
}

Foi desenvolvida a arilação de Heck, regiosseletiva e altamente eficiente, de 3-vinil-6,7dimetoxicumarina, para a obtenção de 3 -estiril cumarinas com bons a excelentes rendimentos. $\mathrm{O}$ aumento da conjugação nos produtos sintetizados reflete na absorvância, revelando todos desvios batocrômicos pronunciados e efeitos hipercrômicos.

Regioselective and highly efficient Heck arylation of 3-vinyl-6,7-dimethoxycoumarin has been developed to afford 3-styryl coumarins in good to very high yields. The increase in conjugation reflects on the absorbance of the synthesized compounds revealing all pronounced bathochromic shifts and hyperchromic effects.

Keywords: 3-vinyl coumarin, 3-styryl coumarin, Heck reaction, palladium, fluorescent dyes

\section{Introduction}

Coumarins (or benzopyranones), whether naturally occurring or synthetic, have attracted the interest of the scientific community due to their broad pharmacological activities, ${ }^{1}$ such as antiprotozoal, ${ }^{2}$ anticancer,${ }^{3}$ antibacterial, ${ }^{4}$ among others. ${ }^{5}$ Depending on the nature and substitution pattern, coumarins show exceptional optical properties, ${ }^{6}$ as they constitute the largest class of fluorescent dyes, ${ }^{7}$ widely used as emission layers in organic light-emitting diodes (OLED), ${ }^{8}$ optical brighteners, ${ }^{9}$ nonlinear optical chromophores, ${ }^{10}$ fluorescent whiteners, ${ }^{11}$ fluorescent labels and probes for physiological measurement ${ }^{12}$ and more recently, in labelling ${ }^{13}$ and caging..$^{14}$ Developments from the last decade show that the introduction of appropriated substituents into the coumarin ring contribute to structures with improved photophysical and spectroscopic proprieties..$^{15}$ Many articles dealing with their synthesis, reactivity and spectral profile have been published. In particular, it seems that the presence of an aryl or heteroaryl moiety on the 3-position of the coumarinic system induces specific activities. ${ }^{16}$ We have recently reported a particularly useful, easy and concise

*e-mail: amlp@uevora.pt synthesis of diversified 3-aryl coumarin using Heck coupling reactions between coumarin and arylhalides. ${ }^{17}$ One can anticipate that the extension of the p-delocalized system will lead to compounds showing a more promising fluorescent behavior. In relation to this, the introduction of an unsaturated fragment between the coumarin ring and the aromatic group attached to the 3-position appears to be an exciting task for which the Heck reaction is a suitable tool. Previously, ${ }^{18}$ the palladium-catalysed insertion of 3-bromocoumarin into a number of alkenes, cycloalkenes and alkynes was already tried. More recently the approach of Bäuerle and co-workers ${ }^{19}$ for the parallel synthesis of a 3-substituted coumarin compounds library rely also on palladium catalyzed reaction between 3-bromocoumarin and appropriated arylvinyl substrates. This work is however limited to the unsubstituted 3-bromocoumarin or to nitrogen substituted ones. A similar approach was used by Kirsch and co-workers ${ }^{20}$ for the preparation of oxygen substituent 3-styryl coumarins. The presence of oxygen substituent groups in coumarins is promising in relation to likely biological activity. Starting with 6,7-dihydroxycoumarin (esculetin) a natural product with high biological activity ${ }^{4}$ we propose to use Heck coupling reaction into the esculetin derivative, 3-vinyl-6,7-dimethoxycoumarin and 3-bromo-6,7dimethoxycoumarin, to afford 3 -styryl coumarins. 


\section{Results and Discussion}

The strategy for the synthesis of 3-styryl substituted coumarins (1a-d) was outlined by two different synthetic routes via Heck coupling reactions (Scheme 1): route A, the halogenated counterpart is the coumarin (2) and route B, the vinylcoumarin (3) is the substrate for the insertion reaction of aryl halides.

To carry out the synthesis of 3-styryl coumarins the synthesis of 3-bromo-6,7-dimethoxycoumarin ${ }^{21}$ (2) was essential which was accomplished with oxone ${ }^{22}$ and $\mathrm{HBr}$ on reaction with 6,7-dimethoxicoumarin (4) (Scheme 2). Compound 2 was obtained in high yield (96\%). The 6,7-dimethoxycoumarin (4) was easily obtained by methylation with diazomethane of 6,7-dihydroxycoumarin (esculetin). Subsequent Suzuki cross-coupling reaction of 2 with potassium vinyltrifluoroborate ${ }^{23}$ allowed 6,7-dimethoxy-3-vinylcoumarin (3), the scaffold used on route $\mathrm{B}$, to be obtained in $95 \%$ yield (Scheme 2).

With both substrates in hand we endeavor a set of palladium coupling reactions. To substrate $\mathbf{2}$, were applied several Heck catalyst systems including those referred in literature ${ }^{18-20}$ but the results were as discouraging as those here presented in Table 1 (route A). These results are consistent with the reactivity of coumarins at the 3-position that is largely dependent on the substitution pattern as any change on the arrangement of substituents leads to unpredictable results. Comparing both results obtained in route A and B we found that route B (although involving one more step) provide us with a more clean reaction which facilitates products isolation and purification allowing the 3 -styrylcoumarins to be obtained in higher yields (Table 1).
Also, and as expected, iodo halides gave a better result, with an increment of approximately 3 fold more than bromo halides (Table 1) which reflects the easiness of the oxidative addition of ArI to the palladium(0) complex. ${ }^{24}$

Table 1. Yields of 3-styrylcoumarins (1)

\begin{tabular}{lccc}
\hline \multirow{2}{*}{ Compound } & route A / $\%$ & \multicolumn{2}{c}{ route B / \% } \\
\cline { 3 - 4 } 1a & 85 & 25 & $\mathrm{X}=\mathrm{I}$ \\
$\mathbf{1 b}$ & 10 & 37 & 88 \\
1c & 24 & 32 & 96 \\
1d & trace & 15 & 90 \\
\hline
\end{tabular}

The method proved to have applicability as varying $R$ with electron-withdrawing and electron-donating groups (Scheme 1) diverse compounds could be synthesized in a straightforward procedure. The electronic delocalization induced by the methoxy groups at the 6 and 7-positions allows the coumarin to have a distinctive behavior for the Heck coupling reactions which enhances this work and make it very useful for this particular family of compounds. Likewise this work presents an interesting approach to generate 3-vinyl coumarins as it relies in natural available substrates, which complements our recently reported procedure which is dependent on the availability of 2-hydroxy aldehydes..$^{25}$ Also both highlight the importance of 3-vinyl coumarins for the synthesis of diverse 3-styryl coumarins. The Heck catalytic system involving $\mathrm{CH}_{3} \mathrm{CO}_{2} \mathrm{Ag}$ as sequestering agent of halide, indicates a cationic mechanism for the coordinationinsertion step, i.e., the coordination of 3 should take place<smiles>[R]c1ccc(C=C)cc1</smiles><smiles>[X]c1ccc([R])cc1</smiles>
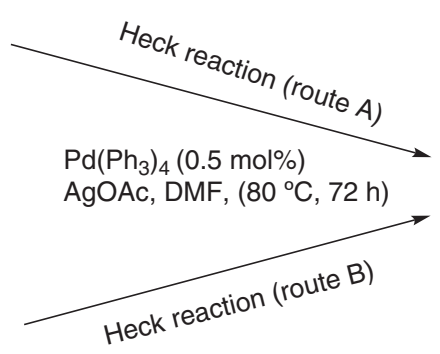<smiles>[R]c1ccc(/C=C/c2cc3cc(OC)c(OC)cc3oc2=O)cc1</smiles>

Scheme 1. Synthesis of 3-styryl coumarins (1) by Heck reaction through halogenated coumarins (route A) and vinyl coumarins (route B).<smiles>COc1cc2ccc(=O)oc2cc1OC</smiles><smiles>COc1cc2cc(Br)c(=O)oc2cc1OC</smiles>

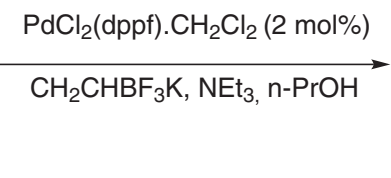<smiles>C=Cc1cc2cc(OC)c(OC)cc2oc1=O</smiles>

Scheme 2. Synthesis of 3-bromo-6,7-dimethoxycoumarin (2) and 3-vinyl-6,7-di-methoxycoumarin (3). 


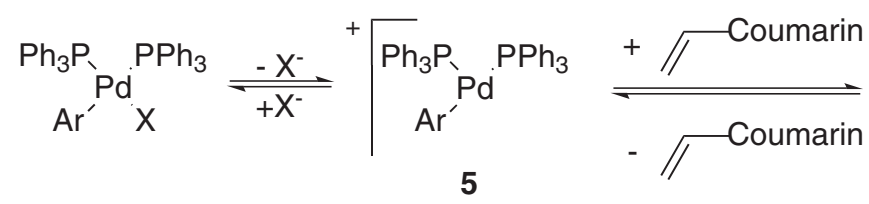

Scheme 3. Proposed mechanism for the coordination-insertion step (route B)

via dissociation of the anionic ligand $\left(\mathrm{X}^{-}\right)$, and a cationic palladium(II) complex $\mathbf{6}$ is formed (Scheme 3). ${ }^{24}$

The reactivity of the cationic complex $\mathbf{5}$ depends on the charge density of the unsaturated system reacting faster with electron rich ones which is the case of the vinyl coumarin 3 (poor $\pi$-acceptors and good $\sigma$-donors). The faster formation of $\mathbf{6}$ will account for the success of the methodology. In both cases the reaction is regioselective and the 2'-arylethenyl coumarins are obtained exclusively. Changing reaction conditions as the amount of catalyst, the base and the solvent revealed that the system 6,7-dimethoxy-3-vinylcoumarin (3) (1.1 equiv.) / aryl halide (1.0 equiv.) / $\mathrm{Pd}\left(\mathrm{PPh}_{3}\right)_{4}(5 \mathrm{~mol} \%) / \mathrm{CH}_{3} \mathrm{CO}_{2} \mathrm{Ag}$ (1.1 equiv.) / DMF / $80^{\circ} \mathrm{C} / 72 \mathrm{~h}$, was the most efficient to furnish the desired 3-styryl coumarins $\mathbf{1}$. The increase in conjugation reflects on the absorbance of the synthesized compounds, revealing all pronounced bathochromic shifts and hyperchromic effects (Table 2, Figure 1).

Table 2. Absorption properties of selected coumarins

\begin{tabular}{lcc}
\hline Coumarin & $\lambda_{\max }{ }^{a} / \mathrm{nm}$ & $\varepsilon_{\max }{ }^{a} /\left(\mathrm{L} \mathrm{mol}^{-1} \mathrm{~cm}^{-1}\right)$ \\
\hline $\mathbf{4}$ & 342 & 12580 \\
$\mathbf{2}$ & 356 & 17370 \\
3 & 361 & 20280 \\
1a & 374 & 25120 \\
1b & 408 & 32650 \\
1c & 398 & 29720 \\
1d & 392 & 28880 \\
\hline
\end{tabular}

${ }^{a}$ longest wavelength transition.

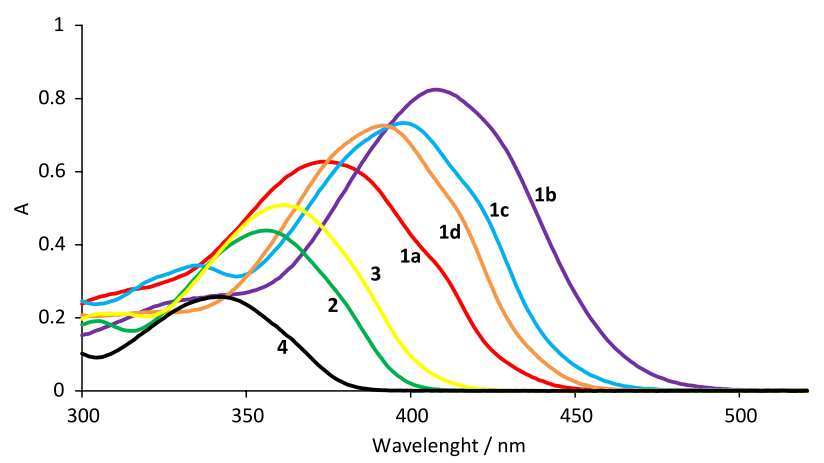

Figure 1. UV-Vis spectra of the 3-styryl coumarins and their precursor $\left(2.5 \times 10^{-5} \mathrm{~mol} \mathrm{~L}^{-1}\right.$, in acetonitrile).

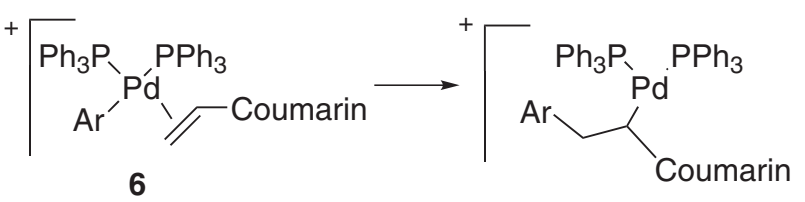

\section{Conclusions}

In summary, with the objective to increase the delocalized p-electron system, new 3-styrylcoumarin derivatives with potential industrial applications, such as new antioxidants and fluorescent chemosensors, were developed by a simple and efficient synthetic strategy involving Heck coupling reactions. The starting material for these reactions, the 6,7-dihydroxycoumarin, is a natural product with high biological activity which can perspective these compounds as promising ones.

\section{Experimental}

All commercial reagents were used as received unless otherwise mentioned. For analytical and preparative thinlayer chromatography, Merck, $0.2 \mathrm{~mm}$ and $0.5 \mathrm{~mm}$ Kieselgel GF 254 percoated were used, respectively. The spots were visualized using UV light and phosphomolybdic acid solution followed by heating. Medium performance liquid chromatography and flash column chromatography were performed using Merck, Kieselgel 60 with 0.063-0.200 mm and $0.040-0.063 \mathrm{~mm}$, respectively. UV-Vis absorption spectra were recorded on a Thermo Electron Corporation (Nicolet Evolution 300) spectrophotometer for solutions in acetonitrile. ${ }^{1} \mathrm{H}$ and ${ }^{13} \mathrm{C}$ NMR spectra were recorded on a Bruker ARX 400 spectrometer at 400 and $100.62 \mathrm{~Hz}$, respectively. ${ }^{1} \mathrm{H}$ shifts are reported relative to internal TMS. Carbon shifts are given relative to the ${ }^{13} \mathrm{C}$ signal of $\mathrm{CDCl}_{3}(\delta 77.0 \mathrm{ppm})$ or $\left(\mathrm{CD}_{3}\right)_{2} \mathrm{CO}(\delta 29.8 \mathrm{ppm})$ as reference. Mass spectra were recorded at the Laboratório de Análises, Requimte, Faculdade de Ciências e Tecnologia, Universidade Nova de Lisboa using a Micromass GC-TOF apparatus and the high resolution mass spectra were recorded at the Mass Spectrometry Unit at the University of Santiago de Compostela, Spain. The electron impact mass spectra were recorded using a magnetic Micromass Autospec apparatus.

Procedure for the bromination of 6,7-dimethoxycoumarin with oxone ${ }^{\circledR}$ and $\mathrm{HBr}$

To a mixture of 6,7-dimethoxycoumarin $(619 \mathrm{mg}$, $3.0 \mathrm{mmol}, 1.0$ equiv. $)$ and oxone ${ }^{\circledR}(1.29 \mathrm{~g}, 4.2 \mathrm{mmol})$ 
in $\mathrm{CH}_{2} \mathrm{Cl}_{2}(12 \mathrm{~mL})$ was added $2 \mathrm{~mol} \mathrm{~L}{ }^{-1} \mathrm{HBr}(3.3 \mathrm{~mL}$, $6.6 \mathrm{mmol}$ ) in one portion resulting a dark red colored solution. After stirring $16 \mathrm{~h}$ at r.t., the color disappeared and $\mathrm{Et}_{3} \mathrm{~N}(1.25 \mathrm{~mL}, 9.0 \mathrm{mmol})$ was added cautiously. After stirring for further $1 \mathrm{~h}$, the reaction mixture was diluted with $\mathrm{CH}_{2} \mathrm{Cl}_{2}$, and washed with $\mathrm{H}_{2} \mathrm{O}$. The organic layer was dried $\left(\mathrm{Na}_{2} \mathrm{SO}_{4}\right)$, filtered, and concentrated under vacuum. The residue was purified by flash column chromatography on silica gel (230-400 mesh; hexane/ $\mathrm{CH}_{2} \mathrm{Cl}_{2}$ gradient) to yield 3-bromo-6,7-dimethoxycoumarin $(821 \mathrm{mg}, 2.88 \mathrm{mmol}$, $96 \%)$.

Procedure for the Suzuki reaction of 3-bromo-6,7dimethoxycoumarin and potassium vinyltrifluoroborate

Under an nitrogen atmosphere, a mixture of potassium vinyltrifluoroborate $(60 \mathrm{mg}, 0.448 \mathrm{mmol})$, $\mathrm{PdCl}_{2}$ (dppf). $\mathrm{CH}_{2} \mathrm{Cl}_{2}$ (6 mg, $0.007 \mathrm{mmol}$ ), 3-bromo-6,7dimethoxycoumarin (2) (106 mg, $0.373 \mathrm{mmol}$ ), and $\mathrm{NEt}_{3}$ (37.7 mg, $0.373 \mathrm{mmol}$ ) in $\mathrm{n}-\mathrm{PrOH}(6 \mathrm{~mL})$ was stirred at reflux for a period of $15 \mathrm{~min}$. After cooling to r.t., the reaction mixture was diluted with $\mathrm{CH}_{2} \mathrm{Cl}_{2}$, and washed with $\mathrm{H}_{2} \mathrm{O}$. The organic layer was dried $\left(\mathrm{Na}_{2} \mathrm{SO}_{4}\right)$, filtered, and concentrated under vacuum. The residue was purified by flash column chromatography on silica gel (230-400 mesh; $\mathrm{CH}_{2} \mathrm{Cl}_{2}$ /EtOAc gradient) to yield 3-vinyl6,7-dimethoxycoumarin (3) (82 mg, $0.353 \mathrm{mmol}, 95 \%)$.

\section{General procedure for the Heck reactions}

\section{Route A}

Under an nitrogen atmosphere, a mixture of 3-bromo6,7-dimethoxycoumarin (2) (285 mg, $1.0 \mathrm{mmol}, 1.0$ equiv.), styrene derivative (1.1 equiv.), $\mathrm{Pd}\left(\mathrm{PPh}_{3}\right)_{4}(5 \mathrm{~mol} \%)$, and $\mathrm{CH}_{3} \mathrm{CO}_{2} \mathrm{Ag}$ (1.1 equiv.) in DMF $(2.0 \mathrm{~mL})$ was stirred at $80^{\circ} \mathrm{C}$ for a period of $72 \mathrm{~h}$. The reaction mixture was diluted with EtOAc, and washed with $\mathrm{H}_{2} \mathrm{O}$. The organic layer was dried $\left(\mathrm{Na}_{2} \mathrm{SO}_{4}\right)$, filtered, and concentrated under vacuum. The residue was purified by flash column chromatography on silica gel (230-400 mesh; hexane/ $\mathrm{CH}_{2} \mathrm{Cl}_{2}$ gradient) to afford the corresponding products.

\section{Route B}

Under an nitrogen atmosphere, a mixture of 3-vinyl6,7-dimethoxycoumarin (3) (232 mg, $1.0 \mathrm{mmol}, 1.1$ equiv.), aryl halide (1.0 equiv.), $\mathrm{Pd}\left(\mathrm{PPh}_{3}\right)_{4}(5 \mathrm{~mol} \%)$, and $\mathrm{CH}_{3} \mathrm{CO}_{2} \mathrm{Ag}$ (1.1 equiv.) in DMF $(2.0 \mathrm{~mL})$ was stirred at $80^{\circ} \mathrm{C}$ for a period of $72 \mathrm{~h}$. The reaction mixture was diluted with EtOAc, and washed with $\mathrm{H}_{2} \mathrm{O}$. The organic layer was dried $\left(\mathrm{Na}_{2} \mathrm{SO}_{4}\right)$, filtered, and concentrated under vacuum. The residue was purified by flash column chromatography on silica gel (230-400 mesh; hexane/ $\mathrm{CH}_{2} \mathrm{Cl}_{2}$ gradient) to afford the corresponding products.

\section{3-Vinyl-6,7-dimethoxycoumarin (3)}

${ }^{1} \mathrm{H}$ NMR (400 MHz, $\left.\mathrm{CDCl}_{3}\right): 3.91\left(\mathrm{~s}, 3 \mathrm{H}, \mathrm{OCH}_{3}\right), 3.94$ (s, 3H, $\left.\mathrm{OCH}_{3}\right), 5.41$ (d, 1H, J $11.3 \mathrm{~Hz}, \mathrm{H}-2$ '), 6.11 (d,1H, J 17.6 Hz, H-2"'), 6.69 (dd, 1H, J 17.6, 11.3 Hz, H-1'), 6.82 (s, 1H, H-8), 6.86 (s, 1H, H-5), 7.63 (s, 1H, H-4). ${ }^{13} \mathrm{C} \mathrm{NMR}\left(100 \mathrm{MHz}, \mathrm{CDCl}_{3}\right): 56.3\left(2 \times \mathrm{OCH}_{3}\right), 99.6(\mathrm{C}-8)$, 107.8 (C-5), 111.9 (C-4a), 118.1 (C-2'), 112.0 (C-3), 130.7 (C-1'), 137.7 (C-4), 146.4 (C-6), 149.1 (C-8a), 152.6 (C-7), $160.6(\mathrm{C}-2)$. MS (EI+) m/z (\%): $232[\mathrm{M}]^{+}(100)$.

\section{(E)-6,7-Dimethoxy-3-styrylcoumarin (1a)}

${ }^{1} \mathrm{H}$ NMR (400 MHz, $\left.\mathrm{CDCl}_{3}\right): 3.93$ (s, 3H, $\mathrm{OCH}_{3}$ ), 3.94 (s, 3H, $\mathrm{OCH}_{3}$ ), 6.83 (s, 1H, H-8), 6.88 (s, 1H, H-5), 7.10 (d, 1H, J 16.3 Hz, H-1'), 7.26-7.29 (m, 1H, H-6'), 7.34-7.37 (m, 2H, H-5', H-7'), 7.51-7.53 (m, 2H, H-4', H-8'), 7.54 (d, 1H, J 16.3 Hz, H-2'), 7.73 (s, 1H, H-4). ${ }^{13} \mathrm{C} \mathrm{NMR}\left(100 \mathrm{MHz}, \mathrm{CDCl}_{3}\right): 56.3\left(2 \times \mathrm{OCH}_{3}\right), 99.6(\mathrm{C}-8)$, 107.7 (C-5), 112.3 (C-4a), 122.0 (C-3), 122.4 (C-1'), 126.8 (C-4', C-8'), 128.1 (C-6'), 128.7 (C-5', C-7'), 132.3 (C-2'), 137.1 (C-4, C-3'), 146.5 (C-6), 148.8 (C-8a), 152.5 (C-7), 160.8 (C-2). MS (EI') m/z (\%): $308\left[\mathrm{M}^{+}\right.$(100). HRMS $\left(\mathrm{EI}^{+}\right)$calc. for $\mathrm{C}_{19} \mathrm{H}_{16} \mathrm{O}_{4}[\mathrm{M}]^{+}$308.1049, found 308.1049.

(E)-6,7-Dimethoxy-3-(4-nitrostyryl)coumarin (1b)

${ }^{1} \mathrm{H}$ NMR (400 MHz, $\mathrm{CDCl}_{3}$ ): 3.95 (s, 3H, $\left.\mathrm{OCH}_{3}\right), 3.97$ (s, 3H, $\left.\mathrm{OCH}_{3}\right), 6.86$ (s, 1H, H-8), 6.90 (s, 1H, H-5), 7.20 (d, 1H, J 16.2, H-1'), 7.64 (d, 2H, J 8.8, H-4', H-8'), 7.70 (d, 1H, J 16.2, H-2'), 7.78 (s, 1H, H-4), 8.21 (d, 2H, J 8.8, $\mathrm{H}-5$ ', H-7'). ${ }^{13} \mathrm{C} \mathrm{NMR}\left(100 \mathrm{MHz}, \mathrm{CDCl}_{3}\right): 56.4\left(2 \mathrm{xOCH}_{3}\right)$, 99.6 (C-8), 107.8 (C-5), 112.0 (C-4a), 120.7 (C-3), 124.1 (C-5', C-7'), 127.1 (C-1',C-4', C-8'), 130.0 (C-2'), 139.7 (C-4), 143.7 (C-3'), 146.7 (C-6), 147.0 (C-6'), 149.3 (C-8a), 153.3 (C-7), 160.3 (C-2). MS (EI+) m/z (\%): 353 $[\mathrm{M}]^{+}(48), 323\left[\mathrm{M}-\left(2 \times \mathrm{CH}_{3}\right)\right]^{+}(100)$. HRMS $\left(\mathrm{EI}^{+}\right)$calc. for $\mathrm{C}_{19} \mathrm{H}_{15} \mathrm{NO}_{6}[\mathrm{M}]^{+}$353.0899, found 353.0900.

\section{(E)-6,7-Dimethoxy-3-(4-formylstyryl)coumarin (1c)}

${ }^{1} \mathrm{H}$ NMR (400 MHz, $\left.\mathrm{CDCl}_{3}\right): 3.92\left(\mathrm{~s}, 3 \mathrm{H}, \mathrm{OCH}_{3}\right), 3.95$ $\left(\mathrm{s}, 3 \mathrm{H}, \mathrm{OCH}_{3}\right), 6.84$ (s, 1H, H-8), 6.89 (s, 1H, H-5), 7.20 (d, 1H, J 16.3, H-1'), 7.63 (d, 1H, J 16.3, H-2'), 7.64 (d, 2H, J 7.6, H-4', H-8'), 7.78 (s, 1H, H-4), 7.85 (d, 2H, J 7.6, H-5', H-7'), 9.97 (CHO). $\left.{ }^{13} \mathrm{C} \mathrm{NMR} \mathrm{(100} \mathrm{MHz,} \mathrm{CDCl}_{3}\right)$ : $56.3\left(\mathrm{OCH}_{3}\right), 56.4\left(\mathrm{OCH}_{3}\right), 99.6(\mathrm{C}-8), 107.9(\mathrm{C}-5), 112.1$ (C-4a), 121.1 (C-3), 126.0 (C-1'), 127.1 (C-5', C-7'), 130.1 (C-4', C-8'), 131.0 (C-2'), 135.6 (C-6'), 138.9 (C-4), 143.2 (C-3'), 146.7 (C-6), 149.2 (C-8a), 153.1 (C-7), 160.4 (C-2), 191.5 (CHO). MS (EI+) m/z (\%): $336\left[\mathrm{M}^{+}\right.$(72). HRMS $\left(\mathrm{EI}^{+}\right)$calc. for $\mathrm{C}_{20} \mathrm{H}_{16} \mathrm{O}_{5}[\mathrm{M}]^{+}$336.0998, found 336.1006. 
(E)-6,7-Dimethoxy-3-(4-methoxylstyryl)coumarin (1d)

${ }^{1} \mathrm{H} \mathrm{NMR}\left(400 \mathrm{MHz}, \mathrm{CDCl}_{3}\right): 3.83\left(3 \mathrm{H}, \mathrm{s}, \mathrm{OCH}_{3}\right), 3.93$ $\left(3 \mathrm{H}, \mathrm{s}, \mathrm{OCH}_{3}\right), 3.94\left(3 \mathrm{H}, \mathrm{s}, \mathrm{OCH}_{3}\right), 6.83(1 \mathrm{H}, \mathrm{s}, \mathrm{H}-8), 6.87$ (1H, s, H-5), 6.89 (2H, d, J 8.6, H-5', H-7'), 6.97 (1H, d, $J$ 16.2, H-1'), 7.46 (2H, d, J 8.6, H-4', H-8'), 7.49 (1H, d, $J$ 16.2, H-2'), 7.68 (1H, s, H-4). ${ }^{13} \mathrm{C}$ NMR (100 MHz, $\left.\mathrm{CDCl}_{3}\right): 55.3\left(\mathrm{OCH}_{3}\right), 56.3\left(2 \mathrm{xOCH}_{3}\right), 99.6(\mathrm{C}-8), 107.6$ (C-5), 112.4 (C-4a), 114.1 (C-5', C-7'), 120.2 (C-1'), 122.2 (C-3), 128.1 (C-4', C-8'), 129.9 (C-3'), 131.8 (C-2'), 136.1 (C-4), 146.5 (C-6), 148.6 (C-8a), 152.3 (C-7), 159.7 (C-6’), 160.9 (C-2). MS (EI') m/z (\%): $338[\mathrm{M}]^{+}$(100). HRMS $\left(\mathrm{EI}^{+}\right)$calc. for $\mathrm{C}_{20} \mathrm{H}_{18} \mathrm{O}_{5}[\mathrm{M}]^{+} 338.1154$ found 338.1156 .

\section{Supplementary Information}

Supplementary data is available free of charge at http://jcbs.sbq.org.br as PDF file.

\section{Acknowledgments}

Thanks are due to the Fundação para a Ciência e Tecnologia (Portugal), for partial financial support FCOMP-01-0124-FEDER-007448.

\section{References}

1. Riveiro, M. E.; De Kimpe, N.; Moglioni, A.; Vazquez, R.; Monczor, F.; Shayo, C.; Davio, C.; Curr. Med. Chem. 2010, 17, 1325; Hoult, J. R. S.; Paya, M.; Gen. Pharmacol. 1996, 27, 713.

2. Pierson, A. J.; Dumetre, T.; Hutter, S.; Delmas, F.; Laget, M.; Finet, J. P.; Azas, N.; Combes, S.; Eur. J. Med. Chem. 2010 45, 864.

3. Combes, S.; Barbier, P.; Douillard, S.; McLeer-Florin, A.; Bourgarel-Rey, V.; Pierson, J. T.; Fedorov, A. Y.; Finet, J. P.; Boutonnat, J.; Peyrot, V.; J. Med. Chem. 2011, 54, 3153; Musa, M. A.; Cooperwood, J. S.; Khan, M. O. F.; Curr. Med. Chem. 2008, 15, 2664; Kostova, I.; Curr. Med. Chem. Anti Canc. Agents 2005, 5, 29.

4. Chin, Y. P.; Huang, W. J.; Hsu, F. L.; Lin, Y. L.; Lin, M. H.; Arch. Pharm. 2011, 344, 386.

5. Dayam, R.; Gundla, R.; Al-Mawsawi, L. Q.; Neamati, N.; Med. Res. Rev. 2008, 28, 118; Carotti, A.; Altomare, C.; Catto, M.; Gnerre, C.; Summo, L.; Marco, A. De; Rose, S.; Jenner, P.; Testa, B.; Chem. Biodivers. 2006, 3, 134; Thuong, P. T.; Hung, T. M.; Ngoc, T. M.; Ha, D. T.; Min, B. S.; Kwack, S. J.; Kang, T. S.; Choi, J. S.; Bae, K.; Phytother. Res. 2010, 24, 101; Kabeya, L. M.; Marchi, A. A. de; Kanashiro, A.; Lopes, N. P.; Silva, C. da; Pupo, M. T.; Lucisano-Valima, Y. M.; Bioorg. Med. Chem. 2007, 15, 1516; Jung, J. C.; Park, O. S.; Molecules 2009, 14, 4790 .
6. Kuznetsova, N. A.; Kaliya, O. L.; Russ. Chem. Rev. 1992, 61, 1243.

7. Jones, G.; Jackson, W. R.; Choi, C.; Bergmark, W. R.; J. Phys. Chem. 1985, 89, 294; Jagtap, A. R.; Satam, V. S.; Rajule, R. N.; Kanetkar, V. R.; Dyes Pigm. 2009, 82, 84; Wagner, B. D.; Molecules 2009, 14, 210; Katerinopoulos, H. E. Curr. Pharm. Des. 2004, 10, 3835.

8. Lee, M. T.; Yen, C. K.; Yang, W. P.; Chen, H. H.; Liao, C. H.; Tsai, C. H.; Chen, C. H.; Org. Lett. 2004, 6, 1241; Swanson, S. A.; Wallraff, G. M.; Chen, J. P.; Zhang, W. J.; Bozano, L. D.; Carter, K. R.; Salem, J. R.; Villa, R.; Scott, J. C.; Chem. Mater. 2003, 15, 2305; Chang, C. H.; Cheng, H. C.; Lu, Y. J.; Tien, K. C.; Lin, H. W.; Lin, C. L., Yang, C. J.; Wu, C. C.; Org. Electron. 2010, 11, 247; Yu, T. Z.; Zhang, P.; Zhao, Y. L.; Zhang, H.; Meng, J.; Fan, D. W.; Org. Electron. 2009, 10, 653.

9. Dorlars, A.; Schellhammer, C. W.; Schroeder, J.; Angew. Chem., Int. Ed. 1975, 14, 665; Kido, J.; Iizumi, Y.; Appl. Phys. Lett. 1998, 73, 2721; Annapurna, D.; Reddy, Y. D.; Somayajulu, V. V.; J. Indian Chem. Soc. 1982, 59, 977.

10. Moylan, C. R.; J. Phys. Chem. 1994, 98, 13513; Painelli, A.; Terenziani, F.; Synth. Met. 2001, 124, 171; Benight, S. J.; Johnson, L. E.; Barnes, R.; Olbricht, B. C.; Bale, D. H.; Reid, P. J.; Eichinger, B. E.; Dalton, L. R.; Sullivan, P. A.; Robinson, B. H.; J. Phys. Chem. B 2010, 114, 11949.

11. Wilze, K. A.; Johnson, A. K.; Handbook of Detergents, Chemistry, Production, and Application of Fluorescent Whitening Agents, Part F; CRC Press, Taylor \& Francis: Boca Raton, 2007.

12. Kim, J. H.; Kim, H. J.; Kim, S. H.; Lee, J. H.; Do, J. H.; Kim, H. J.; Lee, J. H.; Kim, J. S.; Tetrahedron Lett. 2009, 50, 5958; Sheng, R. L.; Wang, P. F.; Gao, Y. H.; Wu, Y.; Liu, W. M.; Ma, J. J.; Li, H. P.; Wu, S. K.; Org. Lett. 2008, 10, 5015; Kim, H. J.; Park, J. E.; Choi, M. G.; Ahn, S.; Chang, S. K.; Dyes Pigm. 2010, 84, 54; Lin, W. Y.; Yuan, L.; Cao, X. W.; Tan, W.; Feng, Y. M.; Eur. J. Org. Chem. 2008, 4981; Jung, H. S.; Kwon, P. S.; Lee, J. W.; Kim, J. I.; Hong, C. S.; Kim, J. W.; Yan, S. H.; Lee, J. Y.; Lee, J. H.; Joo, T.; Kim, J. S.; J. Am. Chem. Soc. 2009, 131, 2008.

13. Goddard, J.-P.; Reymond, J.-L.; Trends Biotechnol. 2004, 22, 363; Heiner, S.; Detert, H.; Kuhn, A.; Kunz, H.; Bioorg. Med. Chem. 2006, 14, 6149.

14. Mayer, G.; Heckel, A.; Angew. Chem., Int. Ed. 2006, 45, 4900; Geißler, D.; Antonenko, Y. N.; Schmidt, R.; Keller, S.; Krylova, O. O.; Wiesner, B.; Bendig, J.; Pohl, P.; Hagen, V.; Angew. Chem., Int. Ed. 2005, 44, 1195; Yu, H.; Li, J.; Wu, D.; Qiu, Z.; Zhang, Y.; Chem. Soc. Rev. 2010, 39, 464; Pinheiro, A. V.; Baptista, P.; Lima, J. C.; Nucleic Acids Res. 2008, 36, e90.

15. Mizukami, S.; Okada, S.; Kimura, S.; Kikuchi, K.; Inorg. Chem. 2009, 48, 7630; Hara, K.; Kurashige, M.; Dan-oh, Y.; Kasada, C.; Shinpo, A.; Suga, S.; Sayama, K.; Arakawa, H.; New J. Chem. 2003, 27, 783; Camur, M.; Bulut, M.; Kandaz, M.; Guney, O.; Supramol. Chem. 2009, 21, 624. 
16. Matos, M. J.; Vina, D.; Quezada, E.; Picciau, C.; Delogu, G.; Orallo, F.; Santana, L.; Uriarte, E.; Bioorg. Med. Chem. Lett. 2009, 19, 3268; Matos, M. J.; Vina, D.; Picciau, C.; Orallo, F.; Santana, L.; Uriarte, E.; Bioorg. Med. Chem. Lett. 2009, 19, 5053.

17. Martins, S.; Branco, P. S.; de la Torre, M. C.; Sierra, M. A.; Pereira, A.; Synlett 2010, 2918.

18. Mitra, A. K.; De, A.; Karchaudhuri, N.; Mitra, J.; J. Chem. Res., Synop. 1998, 766.

19. Schiedel, M.-S.; Briehn, C. A.; Bäuerle, P.; Angew. Chem., Int. Ed. 2001, 40, 4677.

20. Valente, S.; Bana, E.; Viry, E.; Bagrel, D.; Kirsch, G.; Bioorg. Med. Chem. Lett. 2010, 20, 5827.
21. King, F. E.; Housley, J. R.; King, T. J.; J. Chem. Soc. 1954, 1392.

22. Kim, K. M.; Park, I. H.; Synthesis 2004, 2641.

23. Molander, G. A.; Rivero, M. R.; Org. Lett. 2002, 4, 107; Molander, G. A.; Figueroa, R.; Aldrichim. Acta 2005, 38, 49.

24. Cabri, W.; Candiani, I.; Acc. Chem. Res. 1995, 28, 2.

25. Gordo, J.; Avó, J.; Parola, A. J.; Lima, J. C.; Pereira, A.; Branco, P.; Org. Lett. 2011, 13, 5112.

Submitted: November 3, 2011 Published online: February 23, 2012 